\title{
TV/Series
}

$6 \mid 2014$

Écho et reprise dans les séries télévisées (III) : de la métafiction à la transmédialité

\section{Continuité, canonicité et complétude dans Doctor Who}

\section{Florent Favard}

\section{(2) OpenEdition Journals}

Édition électronique

URL : http://journals.openedition.org/tvseries/327

DOI : $10.4000 /$ tvseries.327

ISSN : 2266-0909

Éditeur

GRIC - Groupe de recherche Identités et Cultures

Référence électronique

Florent Favard, «Continuité, canonicité et complétude dans Doctor Who », TV/Series [En ligne], 6 | 2014, mis en ligne le 01 décembre 2014, consulté le 20 avril 2019. URL : http://journals.openedition.org/ tvseries/327 ; DOI : 10.4000/tvseries.327

\section{(c) (i) (9)}

TV/Series est mis à disposition selon les termes de la licence Creative Commons Attribution - Pas d'Utilisation Commerciale - Pas de Modification 4.0 International. 


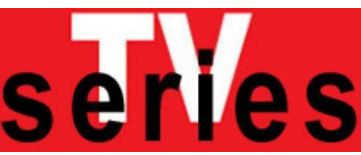

\section{Continuité, canonicité et complétude dans Doctor Who}

Florent FAVARD

Cet article explore le rapport complexe à la continuité qu'entretient la série britannique Doctor Who (BBC One, 1963-1989, 2005-), en se penchant sur sa multi-auctorialité d'un point de vue diachronique puis synchronique, et en détaillant un monde fictionnel dispersé sur de multiples supports suivant un processus qui ne se conforme pas à la définition stricte du transmedia storytelling. La période de production la plus récente du programme (depuis 2010) est aussi analysée, en ce qu'elle révèle une tension entre respect et reconfiguration de la continuité. Les degrés de canonicité des diverses œuvres liées à la série-mère, la réception par les publics et les processus de production du programme sont passés en revue afin de dresser le portrait le plus complet d'une œuvre transmédiatique qui trouve sa dynamique dans l'ellipse via le procédé de complétude rétroactive, dans un développement qui évoque le rhizome plutôt que l'arbre, et dans un renouvellement constant et fluide de ses enjeux esthétiques et économiques.

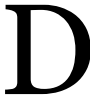

ans l'une des dernières séquences de The Day of the Doctor (7.14), le Docteur éponyme, extraterrestre à l'apparence humaine voyageant à travers l'espace et le temps, vient à la rescousse de sa planète natale, Gallifrey, prise d'assaut par les belliqueux Daleks. Étant capable de se régénérer pour vivre des milliers d'années sous des visages différents, il appelle à l'aide toutes ses incarnations, mises en scène via l'utilisation d'images d'archives. Sont présents les sept Docteurs de la série dite « classique » (BBC One, 1963-1989), le Huitième, issu du téléfilm américano-britannique de 1996, et les trois Docteurs de la nouvelle série (BBC One, 2005-), qui se veut la continuation de la précédente. Deux autres incarnations font leur première apparition dans cet épisode. L'une, interstitielle, entre le Huitième et le Neuvième, complique la biographie du Docteur. L'autre, future, permet de présenter aux spectateurs le prochain interprète du rôle-titre, Peter Capaldi, alors même que le Docteur joué par Matt Smith ne se régénèrera que plus tard dans la saison.

Cet épisode événement, mêlant le passé, le présent et le futur du programme tout en le révisant, vient à point nommé : The Day of the Doctor, diffusé le 23 novembre 2013, célèbre les 50 ans de la série britannique Doctor Who. La longévité du Docteur n'a d'égale que la pérennité du programme qui, malgré une suspension ${ }^{1}$ dans les années 1990, a développé un univers fictionnel dense et complexe s'étendant

${ }^{1}$ Le vocabulaire de la $\mathrm{BBC}$ a toujours été nébuleux concernant l'arrêt de la série classique en 1989, mais elle n'a jamais été annulée, la chaîne laissant espérer une reprise du programme par une autre société de production externe à la BBC. Voir James Chapman, Inside the TARDIS: The Worlds of Doctor Who, Londres, New York, IB Tauris, 2013 (édition révisée), p. 172. 
via des séries dérivées, des aventures audio ${ }^{2}$, des comics, des romans et des jeux vidéo. Mais ce que révèlent aussi The Day of the Doctor, et plus largement la période comprise entre 2010 et 2013, est un rapport plus que jamais ambigu face à la continuité d'un programme qui a été, et est encore, le fruit de nombreuses influences et figures auctoriales, et dont la politique transmedia vise l'expansion plutôt que la cohérence. Au-delà de l'unfolding text perçu par John Tulloch et Manuel Alvarado3, Doctor Who s'apparente à un texte-rhizome ${ }^{4}$, dont la série principale est parfois, selon le point de vue, une racine parmi d'autres, tout en restant la plus visible aux yeux des différents publics.

La présente analyse vise à détailler cette continuité problématique et à explorer les moyens mis en œuvre par le texte et par la «marque» (brand) pour négocier la multi-auctorialité du programme, une politique transmedia aux nombreuses ramifications et, enfin, son monde fictionnel hétérogène.

\section{Un programme en constante régénération}

À l'image de son principal protagoniste, la série Doctor Who a souvent changé de visage au fil des décennies. La régénération du Docteur est d'ailleurs le marqueur le plus visible, lorsqu'il s'agit de catégoriser la série en ères successives, d'une activité menée aussi bien par les fans que par les universitaires, comme le note Paul Booth. Dans son article dédié à la périodisation de Doctor $W h o^{5}$, Booth détaille les nombreux autres critères qui peuvent être retenus, liés au contexte de création, de production et de diffusion du programme. Autre élément prépondérant: la succession des producteurs et des scénaristes en chef, figures auctoriales de la série - que l'on pourrait qualifier, à la suite de François Ronan-Dubois, de " primo-producteurs ${ }^{6}$ ».

Ces primo-producteurs - et productrices, dans le cas de Verity Lambert par exemple - confèrent, de par leur influence, une " production signature » aux épisodes qu'ils supervisent ${ }^{7}$ : un ton, des

2 Diffusées à la radio ou directement disponibles à la vente.

3 John Tulloch, Manuel Alvarado, Doctor Who: The Unfolding Text, New York, Saint Martin's Press, 1983.

4 Gilles Deleuze, Félix Guattari, Capitalisme et schizophrénie: Tome 2, Mille plateaux, Paris, Éditions de Minuit, 1980.

5 Paul Booth, "Periodising Doctor Who », Science fiction film and television, 7.2, 2014, p. $195-215$.

6 « [...] le canon sépare implicitement les primo-producteurs, régies légitimes des discours, des producteurs seconds qui, comme les fans, font vivre l'univers étendu sans nécessairement respecter ses limites justement canoniques. Les primo-producteurs sont par exemple en mesure de légitimer des manipulations thématiques et narratives de la fiction [...]. » François Ronan-Dubois, " Auteurs, régies discursives et primo-producteurs ", Contagions, consulté le 3 septembre 2014.

7 On consultera John Tulloch, Henry Jenkins, Science Fiction Audiences: Watching Doctor Who and Star Trek, Londres, New York, Routledge, 1995. 
thématiques qui permettent de délimiter, par exemple, la période "horreur gothique" sous la direction du producteur Philip Hinchcliffe, entre 1974 et 1977. Une telle catégorisation est d'ailleurs au centre de la méthodologie de l'ouvrage de James Chapman, Inside the TARDIS. Paul Booth explique cependant que toute catégorisation du programme sera toujours subjective, dépendante du contexte dans lequel elle est formulée, et proposera une typologie plutôt qu'une définition. Il suggère d'ailleurs de voir Doctor Who comme un " indefinable text ${ }^{8}$ ".

La succession des primo-producteurs, critère relatif dans le cadre d'une catégorisation des ères du programme, permet toutefois d'analyser son évolution dans une perspective diachronique. Le monde fictionnel de Doctor Who porte la trace des différentes innovations, reconfigurations voire révisions narratives apportées par les différentes équipes de production successives, et motivées par les contextes économiques, sociaux et culturels. La faculté de régénération du Docteur, par exemple, est un mécanisme inventé dans l'urgence pour justifier la transition entre le premier interprète du rôle-titre, William Hartnell, et son successeur Patrick Troughton, dans The Tenth Planet (saison 4, segment ${ }^{9}$ ) en 1966. Les Daleks, ennemis mortels du Docteur, voient leur genèse complètement remaniée à mesure qu'ils gagnent en popularité, entre leur introduction dans The Daleks (saison 1, segment 2) et Genesis of the Daleks (saison 12, segment 4). Une modification justifiée intra-diégétiquement lorsque le Quatrième Docteur, explorant le passé, altère les conditions de leur création.

En 2005, le head writer (scénariste en chef) Russel T. Davies et son équipe choisissent de relancer Doctor Who en manifestant, au sein du monde fictionnel, le fossé qui sépare cette continuation de son aînée, la série classique. La Dernière Grande Guerre du Temps a vu périr les Seigneurs du Temps, le peuple du Docteur, dans leur combat effroyable contre les Daleks. Cette reconfiguration permet de resserrer le monde fictionnel autour de la figure du dernier survivant, justifiant les changements thématiques et le rythme narratif de la nouvelle série, et renouvelant le discours pour mieux explorer le contexte culturel et politique des années 2000. Le traumatisme du Docteur est celui du soldat revenu du front, et la destruction (supposée) de sa planète natale, l'équivalent symbolique du gouffre laissé par les attentats du 11 septembre 2001. En 2010, lorsque Steven Moffat succède à Russel T. Davies, son équipe de production décide d'opérer ce que, manque

${ }^{8}$ Booth, op. cit.

${ }^{9}$ Il reste compliqué de choisir une dénomination pour les histoires de la série classique. Au contraire des saisons contemporaines, les series (saisons) de l'époque étaient composées de segments (stories) constituant une histoire complète, chaque segment rassemblant plusieurs épisodes de 25 minutes. Je choisis ici de numéroter la saison, puis le segment, en comptant à partir du début de chaque saison. 
d'une terminologie adéquate, je qualifierai de "reboot localisé », en modifiant certains aspects de l'ère Davies tout en gardant intacte la continuité. Là encore, la refonte de l'identité du programme par ses primo-producteurs est signalée jusque dans le monde fictionnel, où l'Histoire est peu à peu effacée, et où l'univers doit être, littéralement, redémarré (rebooted) par le Docteur - une marque de réflexivité assumée sur laquelle nous reviendrons.

\section{Une mosaïque d'auteur.e.s}

Après avoir défait Davros et retrouvé la Terre dérobée par les Daleks dans un épisode de la nouvelle série, Journey's End (4.13), le TARDIS, le vaisseau du Docteur, ramène notre planète dans le système solaire. À bord, les compagnes et compagnons du Docteur apprennent à cette occasion que le TARDIS n'est pas fait pour être piloté seul (ce que fait le Docteur dans la quasi-totalité des épisodes des deux séries) mais qu'il nécessite un équipage de six personnes. De la même façon, Doctor Who ne se conduit pas seul.

La multi-auctorialité du programme peut paraître moins évidente dans une perspective synchronique ${ }^{10}$. La nouvelle série, tout particulièrement, se trouve dans un état de superposition parfois paradoxal. Depuis son retour en 2005, elle s'est peu à peu construite comme une brand, une marque, une franchise. Un procédé qui, comme le détaille Catherine Johnson, permet à la $\mathrm{BBC}$ de développer un modèle commercial efficace, de distinguer le produit culturel qu'est Doctor Who du reste de sa production, et de gérer les extensions du programme et les produits dérivés ${ }^{11}$. Mais le processus, selon le brand manager de la série, Ian Grutchfield, est d'abord circonstanciel :

Within the BBC (Public Service) there wasn't a strategic decision to manage key brands - it came about more through circumstances and individual people's ideas. [...]

[...] For Doctor Who, [it] came from the Showrunner model of management deployed by the two visionaries at its helm - i.e. Russel T. Davies writer and executive producer and Julie Gardner, head of drama Wales and executive producer ${ }^{12}$.

\footnotetext{
${ }^{10}$ On consultera par exemple Matt Hills, qui distingue lui aussi une multi-auctorialité diachronique (la succession des auteur.e.s dans le temps) et synchronique (multiplicité d'influences sur un épisode ou une saison donnée). Voir Matt Hills, Triumph of a Time Lord, Londres, New York, IB Tauris, 2010, p. 31.

${ }^{11}$ Catherine Johnson, « Doctor Who as Programme Brand ", in New Dimensions of Doctor Who, éd. Matt Hills, Londres, New York, I.B. Tauris, 2013, p. 96. Voir l'article de Johnson, en particulier pour les tensions qu'elle décrit entre l'aspect commercial du branding et la mission de service public de la BBC.

${ }^{12}$ Cité dans Johnson, ibid., p. 100.
} 
Le « showrunner model » (américain) auquel fait référence Grutchfield concerne le duo formé par les executive producers ${ }^{13}$ Russel T. Davies, scénariste reconnu en Grande-Bretagne, notamment pour son travail sur Queers as Folk (Channel 4, 1999-00) et Bob \& Rose (ITV, 2001) ; et Julie Gardner, dont le poste est l'équivalent d'une directrice de la fiction pour l'antenne galloise de la BBC. Davies et Gardner maîtrisent ainsi les aspects artistiques et économiques de Doctor Who, et supervisent la première ère de la nouvelle série ${ }^{14}$, de son retour sur les écrans en 2005 à la passation de pouvoir en 2010. Le duo est en quelque sorte le visage de la franchise, à une époque où la $\mathrm{BBC}$ cherche à produire un contenu de qualité et à s'éloigner de l'image de programme carton-pâte, à la continuité absconse, que possédait la série classique ${ }^{15}$. Ainsi, à une époque où, comme le souligne Roberta Pearson, le nom d'une auteure est plus attractif que le nom de la chaîne qui diffuse le programme ${ }^{16}$,

[...] the positioning of Davies in particular as "author" of the series equally contributes to the construction of a specific brand identity for the series as a quality text created by a "visionary" that could appeal to the fan and non-fan audience ${ }^{17}$.

Ce phénomène est encore plus prégnant durant l'ère Moffat: les executive producers se succédant d'années en années à ses côtés, il apparaît comme une instance auctoriale dominante, presque omnipotente.

Mais si Steven Moffat reste la figure de proue de la série depuis 2010, le paratexte de Doctor Who a toujours insisté sur la multiauctorialité du programme ${ }^{18}$. La série documentaire Doctor Who Confidential (BBC Three, 2005-11), diffusée immédiatement après chaque épisode de la nouvelle série, met en lumière le travail de toute une équipe sous l'égide du head writer : scénaristes, producteurs mais aussi Murray Gold, le compositeur de la bande sonore musicale, les réalisateurs ou encore les spécialistes des effets spéciaux. L'immense majorité des intervenants se réfèrent à Davies, puis à Moffat, comme la

${ }^{13}$ De même que j'utilise showrunner et head writer, je me permets d'employer executive producer puisque le terme n'a pas d'analogue exact dans la langue française (le plus proche étant « producteur délégué »).

${ }^{14} \mathrm{Si}$, en termes de production, l'ère Davies (2005-10) et l'ère Moffat (2010-) se ressemblent, elles se distinguent par leur esthétique, leurs thématiques et leur approche de la continuité. De même que pour la série classique, principale cible des réserves de Paul Booth, il est possible que la catégorisation des ères Davies et Moffat paraisse désuète et grossière dans quelques dizaines d'années ; pour le moment, par manque de recul, cette distinction reste efficace aux yeux des fans et des universitaires.

15 Voir Hills, op. cit., p. 151 et sq.

${ }^{16}$ Roberta Pearson, «The Writer/Producer in American Television », in The Contemporary Television Series, Michael Hammond et Lucy Mazdon (éds.), Édimbourg, Edinburgh University Press, 2005.

${ }^{17}$ Johnson, op. cit., p. 101.

${ }^{18}$ Hills, op. cit., p. 26, 42. 
plus haute autorité ; dans le même temps, Doctor Who Confidential met en avant les scénaristes de chaque épisode, faisant valoir le modèle britannique: loin des writing room américaines où l'écriture est collective, chaque épisode de Doctor Who entame un dialogue entre son propre scénariste et le head writer, qui peut guider, corriger, voire réécrire le script pour apporter plus de cohésion à la saison ${ }^{19}$. Ainsi, dans le Confidential dédié à Victory of the Daleks (5.3) peut-on voir intervenir, en plus de Steven Moffat, Mark Gatiss, scénariste-phare de la série. Gatiss et Moffat sont ici traités à égalité, et c'est bien Gatiss que l'on voit visiter le Cabinet War Room de Churchill, qui a servi d'inspiration pour l'épisode ; c'est son travail de recherche qui est mis en avant.

Si la série classique n'offrait pas autant d'informations sur son contexte de production, le paratexte contemporain met en valeur sa multi-auctorialité. La BBC a produit, dans le cadre du cinquantenaire de Doctor Who en 2013, un docu-fiction destiné à relater la création du programme et ses premières années, jusqu'au remplacement de William Hartnell par Patrick Troughton. Écrit par Mark Gatiss, An Adventure in Space and Time dévoile les coulisses de la BBC en 1963 , et met en scène la multiple paternité/maternité du programme. Si le head of drama Sydney Newman est crédité de l'idée originale, le docufiction insiste sur la position déterminante, mais malaisée, de deux icônes : Verity Lambert, productrice dans un monde d'hommes, qui va sauver le programme malgré des débuts difficiles; et William Hartnell, acteur au fort caractère qui, d'abord dubitatif, va s'accaparer le rôle, mais dont l'interprétation sera marquée par la maladie. An Adventure in Space and Time dévoile aussi les figures moins connues de Waris Hussein, réalisateur des premiers épisodes (quand on sait, aujourd'hui, l'importance de l'esthétique proposée par un épisode pilote) ; et de Peter Brachacki, le designer qui, exaspéré par l’insistance de Lambert, construit la maquette du TARDIS, le vaisseau du Docteur, en deux temps trois mouvements, avec ce qui lui tombe sous la main. La séquence de la maquette cristallise l'image carton-pâte de la série classique, que An Adventure in Space and Time nous présente avec une certaine nostalgie et une évidente fierté, alors que la qualité discutable des effets spéciaux et des décors de la série a été l'un des critères déterminants dans la suspension de Doctor Who en 1989.

Ce que les paratextes contemporains dévoilent, ce sont d'abord des passionnés, et, tout particulièrement en ce qui concerne la nouvelle série, des fans de Doctor Who. Matt Hills détaille le processus qui a poussé de jeunes spectateurs et spectatrices de la série classique à devenir professionnels, souvent par le biais d'activités de fans

19 On consultera notamment Russel T. Davies, Benjamin Cook, The Writer's Tale: The Final Chapter, Londres, BBC Books, 2010. 
(fanzines, fanfiction, etc.) puis via l'univers « étendu » de Doctor Who (écriture de romans, production d'aventures audio, etc.) jusqu'à participer à la résurrection de la série en 2005. Hills note que cette "Doctor Who Mafia » va à l'encontre des thèses initiales de Henry Jenkins, de l'idée d'une séparation stricte entre les primo-producteurs et les fans ${ }^{20}$. Davies, puis Moffat, mais aussi David Tennant, interprète du Dixième Docteur, et Peter Capaldi, du Douzième, s'avouent volontiers fans de la série classique, et ce positionnement de fanturned-gamekeepers ( fans devenus maîtres du jeu », comme les nomme Hills) est valorisé par la communication de la BBC, qui y voit un gage de respect du matériel narratif face aux fans les plus exigeants.

Cependant, le savoir encyclopédique ainsi partagé par les fans et les primo-producteurs aurait un revers : le fanwanking ${ }^{21}$, qui consiste, au sein d'un épisode, à faire d'obscures références au passé du programme pour le seul plaisir des fans, au risque d'aliéner des publics profanes. Matt Hills relativise le phénomène, expliquant que la nouvelle série parvient à faire référence à sa continuité tout en restant accessible aux non-initiés, superposant des niveaux de lecture à densités variables ${ }^{22}$. Il critique du même coup une vision binaire des publics de Doctor Who, entre fans assidus et profanes. La densité du monde fictionnel, la variété des supports sur lesquels le programme se déploie depuis ses débuts, et les degrés de canonicité du matériel narratif à disposition du public, impliquent une approche multimodale des « whovians » : il y a peut-être autant de façon d'être fan de Doctor Who qu'il y a d'auteur.e.s et d'influences affiliées au programme.

\section{Un univers transmedia hétéroclite}

L'univers «étendu » de Doctor Who ne s'apparente pas à du transmedia storytelling au sens strict. Le concept, popularisé par Henry Jenkins ${ }^{23}$, insiste sur la dispersion des éléments fictionnels sur divers médias, mais aussi sur le caractère " unifié » de l'expérience transmédiatique vécue par les publics - un exemple canonique parmi d'autres étant la campagne transmédia de la trilogie Matrix (Andy et

${ }^{20}$ Hills, op. cit., p. 54 et sq.

${ }^{21}$ Pour des raisons qui apparaîtront évidentes, je laisse aux lecteur.ice.s la possibilité de traduire ce terme grossier, popularisé par les fans de Doctor Who dans les années 1980, et qui a très vite pris une connotation négative - certainement parce qu'une partie des fans persiste à penser que les références obscures à la continuité ont été l'un des critères déterminants dans la suspension de la série classique, hypothèse toujours débattue aujourd'hui.

${ }^{22}$ Hills, op. cit., p. 58 et sq.

23 Henry Jenkins, Convergence Culture: Where Old and New Media Collide, New York, NYU Press, 2006. 
Lana Wachowski, 1999, 2003). Les différents éléments, s’ils ne sont pas tous nécessaires à la compréhension de l'histoire, augmentent l'impression de complétude du monde fictionnel et permettent, par exemple, d'explorer des intrigues secondaires que l'œuvre principale esquisse à peine.

Doctor Who est perçu par Lynette Porter comme l'une des plus vieilles licences transmédia ${ }^{24}$; dans le même temps, Neil Perryman explique que les plus anciens éléments «transmédia» de la série seraient aujourd'hui reconnus comme du «franchising », des produits dérivés $^{25}$ - par exemple, les novélisations des épisodes de la série classique, parus entre 1964 et 1991 chez Target Books. Mais l'univers étendu de Doctor Who, présent sur cinq décennies et une multiplicité de supports, ne peut pas être si facilement catégorisé, car il marie des intentions esthétiques et commerciales radicalement différentes selon les éditeurs, et une approche du monde fictionnel qui mène parfois à des contradictions étonnantes.

À la télévision, Doctor Who possède deux séries dérivées, Torchwood (BBC 3, 2, 1, Starz, 2006-2011) et The Sarah Jane Adventures (CBBC, 2007-2011). Elles ont été lancées durant l'ère Davies (toutes deux sont créées et supervisées par Davies et Julie Gardner) et entretiennent des rapports étroits avec la série-mère. Elles possèdent donc le plus haut niveau de canonicité : les événements mis en scène dans les séries dérivées appartiennent au monde fictionnel de Doctor Who sans qu'aucun doute ne soit possible. Le statut des miniséries animées est plus ambigu : The Infinite Quest (CBBC, 2007) et Dreamland (BBC Red Button, 2009) mettent en scène le Dixième Docteur de l'ère Davies mais ne sont jamais directement référencées par la série-mère. En dehors du canon, Scream of the Shalka, minisérie animée diffusée en 2003 sur Internet par la BBC, possède un statut liminal. Elle met en scène un Neuvième Docteur " alternatif ", dont l'existence est invalidée lors du retour de Doctor Who en 2005, lorsque qu'un autre Neuvième Docteur, incarné par Christopher Eccleston, devient de facto le Neuvième Docteur canonique.

Au-delà de la télévision, Doctor Who et ses séries dérivées s'étendent via un réseau de romans, d'aventures audio et de comics. En s'appuyant sur les données de Tardias.wikia, le wiki anglophone le plus riche dédié à la série, on peut dater le début des publications de romans originaux aux années 1980 . Des séries de romans plus denses apparaissent dans les années 1990 chez Big Finish et BBC Books. Elles permettent de découvrir des aventures inédites du Docteur - ou plutôt,

24 Lynnette Porter, Doctor Who, American Influence, Fan Culture and the Spinoffs, Jefferson, Londres, McFarland \& Company, 2012, p. 78.

${ }^{25}$ Neil Perryman, "I AM THE DOCTOR !": Transmedia Adventures in Time and Space », in Doctor Who, The Eleventh Hour, Andrew O'Day (éd.), Londres, New York, IB Tauris, 2014, p. 232. 
des Docteurs. BBC Books, par exemple, propose plusieurs séries. Les Past Doctors Adventures concernent les Docteurs de la série classique, et ne comprennent pas moins de 75 titres parus entre 1997 et 2005. À l'arrivée de la nouvelle série en 2005, la BBC joue de son statut " officiel» de primo-productrice en publiant des séries de romans dédiées à chacun des nouveaux Docteurs, et dont la durée de vie est égale à leur présence sur les écrans. Ainsi, le Neuvième Docteur, qui n'est apparu que dans la première saison de la nouvelle série, apparaît dans six romans publiés en 2005. Entre 2006 et 2010, alors que David Tennant incarne le Dixième Docteur à la télévision, le personnage vit aussi 57 aventures sur le papier entre 2005 et 2009. La lecture des romans n'est pas nécessaire pour comprendre la série télévisée, mais la synchronisation entre diffusion télévisée et publication renforce le lien symbolique entre les deux supports.

La BBC possède depuis 1996 un monopole auparavant détenu par Virgin Books (pour les romans) et Big Finish (pour les aventures audio). Il est intéressant de constater que Big Finish n'a le droit d'utiliser que les Docteurs de la série classique et celui du téléfilm de 1996, laissant à la BBC les Docteurs de la nouvelle série. Big Finish a néanmoins étendu sa gamme en proposant les aventures audio des compagnes et compagnons du Docteur, et notamment de Bernice Summerfield, apparue sur le papier chez Virgin Books en 1992. La canonicité des aventures de Summerfield peut paraitre discutable : elle n'est jamais apparue ni n'a été mentionnée à la télévision, alors même que le nombre d'œuvres la mettant en scène approche la centaine en 2014, et qu'elle interagit avec plusieurs incarnations du Docteur. Summerfield cristallise le caractère rhizomique de l'univers étendu de Doctor Who: pour pallier aux restrictions imposées par la BBC, qui contrôle l'univers transmédia autour de la nouvelle série, les éditeurs affiliés ont étendu l'univers fictionnel dans des directions variées qui, parfois, deviennent presque indépendantes.

Les éléments les plus contradictoires prennent place durant l'interstice ${ }^{26}$ qui sépare la série classique (1989) de la nouvelle série (2005), et qui n'est marqué que par le téléfilm de 1996 (à la continuité discutable, sur laquelle nous reviendrons). Entre 1989 et 1996 notamment, ce ne sont pas moins de trois primo-producteurs qui se disputent le monde fictionnel: Virgin Book (romans), Big Finish (audio) mais aussi Doctor Who magazine, publié mensuellement depuis 1980 (hebdomadaire à sa création en 1979), qui propose des comics mettant en scène le Docteur. À cela s'ajoute l'arrivée de BBC

${ }^{26}$ J'utilise « interstice " pour reprendre l'expression de Russel T. Davies, qui voit Doctor Who comme un programme structuré autour de gaps : la suspension entre 1989 et 2005, mais aussi la disparition de certains épisodes de la série classique. Il considère que ces interstices sont attractifs pour des générations de fans qui vont chercher à les combler et, par là peut-être, à entrer comme lui dans le métier. Cité dans Hills, op. cit., p. 55 
Books en 1996, qui va reprendre en main l'univers en niant, parfois, le travail des autres éditeurs et en imposant sa continuité plus " officielle ${ }^{27}$ ». Un exemple intéressant est proposé par Tardis.wikia : la biographie du Maître (un autre ennemi du Docteur) est rendue chaotique par les différents éditeurs. Virgin Books continue à mettre en scène le Maître dès 1994 et construit autour de lui toute une continuité qui sera invalidée par BBC Books deux ans plus tard, afin de rendre la biographie du personnage plus cohérente avec le téléfilm de 1996. Pendant ce temps, Big Finish, qui doit trouver des interprètes pour ses aventures audio, choisit un acteur qui avait incarné le Maître dans la série classique, avant d'être remplacé. Pour expliquer pourquoi «son » Maître a la voix d'une incarnation que la série classique a enterrée, Big Finish altère la continuité pour le « ramener à la vie ${ }^{28}$.

Si d'autres problèmes similaires apparaissent dans la continuité de l'univers étendu de Doctor Who, il n'en reste pas moins que le texte se prête aux reconfigurations narratives. Non seulement le voyage dans le temps permet d'expliquer ces abrupts changements, mais la structure même du texte, elliptique, autorise toutes les extensions. Dans la série, il est rare que le Docteur et ses compagnons mentionnent l'ordre précis de leurs différents voyages. Il est donc possible d'intercaler à loisir des aventures papier, audio, voire vidéoludiques, entre des épisodes de la série. Si les modifications dans la biographie du Maître, citées plus haut, s'apparentent sans ambiguïté au procédé de la continuité rétroactive, la série repose plus largement sur un principe similaire que je tiens cependant à distinguer : celui de la complétude rétroactive. Doctor Who ne modifie pas tant sa continuité qu'elle l'augmente, remplissant les interstices et changeant le point de vue des publics sur des éléments déjà établis, sans forcément modifier les éléments eux-mêmes. Ainsi du War Doctor, l'incarnation interstitielle que The Day of The Doctor impose en 2013 entre le Docteur du téléfilm et la neuvième incarnation de 2005 : son existence ne brise pas la continuité mais étend la connaissance de la biographie $\mathrm{du}$ Docteur et introduit une subtilité dans le décompte de ses incarnations qui ouvre, de façon logique, la voie à de possibles autres incarnations interstitielles jamais mentionnées auparavant. Plutôt que

${ }^{27}$ Le wiki anglophone le plus dense consacré à Doctor Who, Tardis.wikia, affiche, au-dessus des pages concernant des éléments considérés comme non-canoniques, l'encadré suivant : "You can believe this subject is part of the Doctor Who universe. But we don't. " Le site, s'il affirme son autorité en se fondant sur la continuité proposée par la BBC et ses éditeurs affiliés, reconnaît aux fans le droit de définir leur propre canon. Lynnette Porter, quant à elle, insiste sur la différence entre les séries canon (le matériel narratif proposé par la BBC à la télévision) et les romans et aventures audio, sanctioned (approuvés par la BBC, mais avec un degré de canonicité moindre, ne serait-ce que parce que ces produits culturels, vendus par des tiers, n'entrent pas dans la mission de service public de la BBC). Voir Porter, p. 84. ${ }_{28}$ On consultera sur la page " The Master » (consultée le 3 septembre 2014) l'encadré intitulé « Making Sense of the 1990 » 
d'être une force centripète qui insisterait sur la cohésion de l'univers fictionnel, le transmédia pratiqué par Doctor Who est centrifuge et étend son univers de manière elliptique. Plus que du transmedia storytelling, le dispositif s'apparente à du transmedia branding : il fait vivre la franchise de manière dynamique, créant un univers hétérogène riche en possibilités et qui ne tourne pas en orbite autour de la seule série-mère pour toucher une grande variété de publics.

Matt Hills voit une autre utilité au dispositif transmédia, dans le cas de la série dérivée Torchwood. En plus de changer de chaîne chaque année, le programme a vu sa production et sa narration évoluer au fil du temps. Les deux premières saisons de treize épisodes chacune sont suivies d'une courte troisième saison au ton beaucoup plus sombre, puis d'une quatrième co-produite avec la chaîne américaine Starz. Cette dernière est perçue par une partie des fans comme niant ses origines en exportant les personnages aux États-Unis. Hills détaille le contenu transmédia qui a accompagné la série, et évoque la notion de "fanagement» (gestion des fans par les producteurs) : les romans servent ici à tisser des liens entre les saisons pour « aplanir » l'univers fictionnel et justifier la transition vers l'internationalisation de la série. Ils ont aussi pour but d'apaiser les fans en expliquant les conséquences (invisibles à l'écran) de la mort tragique d'un personnage majeur à la fin de la saison 3, tout en confisquant aux fans la possibilité de remplir ces interstices via leurs fanfictions ${ }^{29}$. Sous couvert de cohérence, ces œuvres dérivées visent, selon Hills, à taire les doléances des fans et à imposer des changements drastiques dans le ton et la configuration du monde fictionnel.

On trouve enfin, au sein de l'univers étendu de Doctor Who des exemples de transmedia storytelling au sens strict du terme, mais ils sont soit discrets (les webisodes précédant certains épisodes, diffusés sur Internet et servant de "préquelle », de prologue), soit infructueux. Les Adventure Games, en ce sens, constituent un semi-échec. Mis à disposition sur Internet par la BBC, ces jeux vidéo accompagnent les saisons 5 et 6 de la nouvelle série, soit le début de l'ère Moffat (2010 et 2011). Ils sont écrits par Phil Ford et James Moran, scénaristes sur Doctor Who, Torchwood et The Sarah Jane Adventures, et mettent en scène le Onzième Docteur et sa compagne d'alors, Amy Pond, doublés par les interprètes de la série, Matt Smith et Karen Gillan. Si les aventures qu'ils mettent en scène ne sont pas mentionnées à la télévision, les Adventure Games sont bien censés se dérouler à des moments spécifiques, entre deux épisodes de la saison 5 ou 6 . Concernant les quatre jeux publiés durant la saison 5, Piers Wenger, executive producer à cette époque, explique :

${ }^{29}$ Matt Hills, "Torchwood's trans-transmedia: Media tie-ins and brand "fanagement" ", in Participations, Vol. 9, No. 2, novembre 2012. 
There aren't 13 episodes of Doctor Who this year. There are $17-$ four of which are interactive. Everything you see and experience within the game is part of the Doctor Who universe.

Neil Perryman détaille les efforts fournis par la BBC pour faire la publicité des jeux et assurer les publics de leur authenticité - jusqu'à rappeler que Steven Moffat en personne, le head writer de la série, a supervisé leur création ${ }^{30}$. Malheureusement, les jeux ne contribuent pas à l'intrigue de la saison 5 (la réécriture de l'Histoire et le reboot de l'univers par le Docteur) ${ }^{31}$ et ne rencontrent pas le succès. L'idée de publier plusieurs saisons de jeux est abandonnée.

Malgré ce revers, il n'est pas étonnant que les Adventure Games aient été publiés durant l'ère Moffat: ils font partie d'un plus vaste projet qui consiste à prendre la mesure de la cohérence de l'univers fictionnel, et à unifier l'expérience des publics après 50 ans d'un transmedia branding hétéroclite, à la fois riche et hasardeux. Ce projet esthétique ne se manifeste pas seulement en termes économiques, puisqu'il impacte jusqu'au monde fictionnel lui-même.

\section{4. «Reboot the universe »}

Comptant parmi les éléments les plus problématiques de la continuité, le téléfilm de 1996 est pourtant conçu comme la première tentative de continuation de la série classique. Coproduction américano-britannique (BBC, Universal, Fox), il s'ouvre avec le Septième Docteur, joué par Sylvester McCoy - qui tenait déjà le rôletitre à la fin de la série classique. Alors qu'il transporte les restes de son ennemi de toujours, le Maître, il est abattu par les membres d'un gang lorsque le TARDIS atterrit à San Francisco en 1999. Le téléfilm met en scène sa régénération, le remplaçant par sa huitième incarnation, sous les traits de l'acteur britannique Paul McGann.

Si ce Huitième Docteur, supervisé en partie par la BBC et faisant directement suite à la dernière incarnation de la série classique, paraît canonique, le téléfilm suggère que le Seigneur du Temps est en réalité à moitié humain, entrant en contradiction avec les éléments connus de sa biographie ${ }^{32}$. De plus, les taux d'audiences décourageants - notamment aux États-Unis - condamnent une possible nouvelle série à une mort prématurée. Le Huitième Docteur devient ainsi l'incarnation à la durée de vie la plus courte à l'écran, 85 minutes.

Mais c'est sans compter sur l'univers étendu, qui s'empare du personnage, justifie ou gomme son ascendance problématique de

\footnotetext{
${ }^{30}$ Perryman, op. cit., p. 232-233.

${ }^{31}$ Perryman, op. cit., p. 236.

${ }^{32}$ Un autre cas de continuité rétroactive : ici un élément clairement établi est modifié.
} 
diverses manières 33 , et lui fait vivre au moins autant d'aventures que ses prédécesseurs : plus de 73 romans chez BBC Books entre 1997 et 2005, et plus de $73^{34}$ audio chez Big Finish dans les diverses collections mettant en scène le Docteur, entre 1999 et 2014. Paul McGann, l'interprète, a autorisé BBC Books à utiliser son image et a joué son propre rôle dans les aventures audio, trouvant là l'occasion de développer le personnage et de laisser sa marque dans la succession des incarnations du Docteur. Bien qu'absent des écrans, le Huitième Docteur est donc, de 1996 à 2005, considéré comme le Docteur " actuel », avant l'arrivée de Christopher Eccleston. La nouvelle série ne montre pas la régénération du Docteur de McGann en celui de Eccleston, mais n'en respecte pas moins le décompte des incarnations, achevant de donner au Huitième Docteur un statut canonique.

À l'occasion du cinquantenaire de la série en 2013, deux webisodes sont diffusés dans les deux semaines qui précèdent l'épisode-événement The Day of the Doctor. Paul McGann reprend, à l'écran, le rôle du Huitième Docteur dans The Night of the Doctor (BBC iPlayer, 14 novembre 2013), alors que le personnage, au seuil de la mort, traverse la régénération qui fait de lui le War Doctor interstitiel, interprété par John Hurt. Avant de « mourir », le Huitième Docteur a l'occasion de mentionner les compagnes et compagnons qui ont vécu de nombreuses aventures avec lui, mais ne cite que celles et ceux issus des aventures audio Big Finish, ignorant les personnages rencontrés dans les romans de BBC Books ou les comics. Ce webisode canonique écrit par Steven Moffat est le premier à mentionner les productions Big Finish et à leur accorder le plus haut statut canonique, un choix qui sonne comme la reconnaissance du travail de l'éditeur.

Cette référence à l'univers étendu de Doctor Who peut paraitre contradictoire au sein d'une ère qui, depuis 2010, prend ses distances avec la continuité sur la direction du head writer Steven Moffat. La saison 5 de la nouvelle série marque en effet une rupture unilatérale avec les saisons précédentes: le casting est renouvelé dans son intégralité35 avec l'arrivée d'un Onzième Docteur (Matt Smith) et d'une nouvelle compagne, Amy Pond (Karen Gillan); les décors changent

33 Tardis.wikia dénombre l'incertitude (McINTEE, David, Autumn Mist, BBC Books, 1999), des procédés de procréation artificielle (MAGRS, Paul, The Scarlet Empress, BBC Books, 1998), le mensonge éhonté (LEE, Tony, The Forgotten vol. 1-6, IDW Publishing, 2008-09) ou encore la vérité (PARKIN, Lance, The Gallifrey Chronicles, BBC Books, 2005). On notera que trois de ces versions contradictoires cohabitent chez le même éditeur...

34 J'emploie des chiffres approximatifs, me limitant aux aventures les plus canoniques, mais d'autres apparitions peuvent entrer en ligne de compte.

35 Fait rare dans les deux séries, qui ont toujours cherché à conserver un personnage familier pour "contrebalancer» les transitions entre les Docteurs. Voir Pierce Britton, "It's All New Doctor Who: Authorising New Design and Redesign in the Steven Moffat Era ", in Doctor Who, The Eleventh Hour, Andrew O’Day (éd.), Londres, New York, IB Tauris, 2014, p. 149. 
aussi, avec notamment une refonte complète de l'intérieur du TARDIS ; derrière la caméra, Russel $\mathrm{T}$. Davies cède sa place de head writer à Steven Moffat tandis que Piers Wenger et Beth Willis remplacent Julie Gardner au poste de executive producer. Moffat, qui a déjà écrit certains épisodes de l'ère Davies, possède une signature particulière: une ambiance de dark fairy tale («conte de fée sombre »), un penchant pour les paradoxes temporels, la réflexivité et les récits complexes. Une signature qu'il va déployer à l'échelle de la série, faisant notamment des saisons 5 et 6 des ensembles feuilletonnants fonctionnant comme des puzzles ${ }^{36}$.

Ce changement abrupt est manifesté au sein du monde fictionnel dans la saison 5, la première de l'ère Moffat: suite à l'apparition de mystérieuses failles dans l'espace et le temps, le Docteur comprend au fil des épisodes que l'Histoire est effacée petit à petit. Dès Victory of the Daleks (5.3), ce reboot "localisé » (seuls certains éléments sont annulés ou reconfigurés) impacte le monde fictionnel. Le Docteur cherche à convaincre Winston Churchill que les Daleks, malgré les apparences, sont tout sauf ses alliés dans la guerre contre l'oppression nazie. S'ensuit cet échange révélateur :

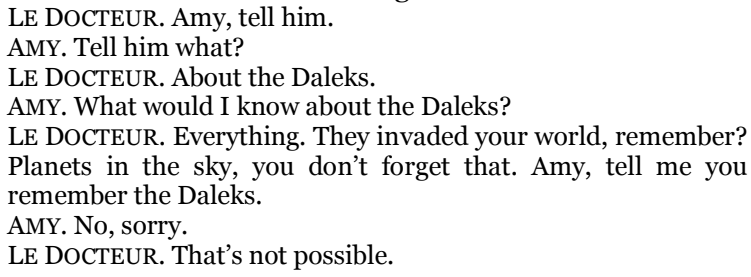

Le Docteur fait ici référence aux événements survenus dans le diptyque The Stolen Earth/Journey's end (4.12, 4.13), lorsque les Daleks ont déplacé la Terre à des années-lumière du système solaire. Le dialogue ci-dessus a trois fonctions. Premièrement, il sert de clin d'œil à la continuité en citant des épisodes de l'ère Davies - seuls les spectateurs les plus assidus comprendront la référence. Moffat indique ici qu'il n'a pas coupé les ponts avec le passé du programme, et que le monde fictionnel reste dans la droite ligne de l'ère Davies. Deuxièmement, la référence permet aux spectateurs profanes d'en découvrir plus sur le monde fictionnel et d'apprendre qui sont les Daleks. Troisièmement, le dialogue permet de souligner l'effacement d'une continuité dont seul le Docteur se souvient, et articule l'arc narratif de la saison. Si le Docteur reboot l'Univers à la fin de la saison, rétablissant (de manière

${ }^{36}$ Concernant l'écriture de Moffat, on consultera notamment Frank Collins, « Monsters Under the Bed: Gothic and Fairy-Tale Storytelling in Steven Moffat's Doctor Who ", in Andrew O’Day, op. cit. 
présumée) la continuité telle que les fans la connaissent, cet éloignement temporaire d'avec les ères précédentes permet à Moffat d'introduire de nouveaux antagonistes et protagonistes, mettant à égalité fans et profanes, faisant de la saison 5 un point d'entrée possible pour de nouveaux publics. Le head writer assume aussi ce processus de déconstruction de la continuité pour mieux imposer sa marque sur Doctor Who.

La saison 7 de la nouvelle série, qui précède le cinquantenaire du programme, est l'occasion d'un nouveau jeu avec la continuité : dans l'épisode qui conclut la saison, The Name of the Doctor (7.14), Clara Oswald, la nouvelle compagne du Docteur, se retrouve douée d'ubiquité et rencontre le Docteur sous toutes ses incarnations via l'utilisation d'images d'archives. La technique n'est pas nouvelle : grâce à des flashbacks ou à la consultation de banques mémorielles, l'apparition des anciennes incarnations à l'écran est courante dans la nouvelle série, et l'ère Moffat en fait un usage marqué à deux reprises : lors du premier épisode du Onzième Docteur, The Eleventh Hour (5.1) et dans The Lodger (5.11). À chaque fois, il s'agit d'établir le personnage joué par Matt Smith comme le dernier d'une longue lignée, alors même que la continuité est temporairement mise en retrait au profit d'une reconfiguration des enjeux narratifs.

The Name of the Doctor a ceci de particulier qu'une interaction est suggérée entre Clara et les Docteurs via la manipulation du son et de l'image ; William Hartnell, le Premier Docteur, semble notamment lui répondre lorsqu'elle l'interpelle. Clara est ici pour empêcher la Grande Intelligence (un autre antagoniste récurrent) de tuer le Docteur à divers moments de son passé : elle a pour tâche de préserver la continuité en la revisitant. C'est d'ailleurs à cette occasion que l'incarnation interstitielle créée par Steven Moffat, et jouée par John Hurt, est aperçue pour la première fois. Clara a sauvé la vie du Docteur et la continuité, mais l'épisode joue aussi sur la complétude rétroactive, révélant ce que le Docteur avait tenu secret : qu'une de ses incarnations ne portant pas le titre de «Docteur » avait participé à la Dernière Grande Guerre du Temps.

Si Paul Booth voit, de façon plus conventionnelle, The Name of the Doctor comme une forme de continuité rétroactive (alors que rien n'y est modifié ou annulé, seulement complété), il dessine une analogie efficace dans son article sur la périodisation de la série, voyant Doctor Who comme une archive. Empruntant à la notion de littérature archontique proposée par Abigail Derecho, il explique que le fonctionnement de Doctor Who comme des wikis qui documentent le programme se fonde sur le principe d'une archive ouverte, en flux constant. Le texte n'est pas figé dans la pierre, mais peut être sans cesse revu et corrigé, étendu, annulé, complété. De plus, il conserve la trace de ces modifications : tout comme une archive, Doctor Who en 
tant que texte n'est pas simplement un artefact mais un processus qui laisse une marge à la reconfiguration, rejoignant ainsi la notion, évoquée par Booth, d'un indefinable text 37 .

L'exemple - ouvrant cette analyse - du sauvetage de la planète Gallifrey par toutes les incarnations du Docteur, dans l'épisode anniversaire The Day of the Doctor, s'inscrit dans une tradition qui date de la série classique : les épisodes à multiples Docteurs, marquant généralement les décennies du programme (The Three Doctors en 1973, The Five Doctors en 1983). Lorsque les Docteurs annulent la destruction de Gallifrey - jouant ici, sans ambiguïté, sur la continuité rétroactive -, il apparaît évident que Moffat se sert de la continuité comme d'une arme à double tranchant : il convoque tous les Docteurs dans une séquence sans précédent pour rendre honneur à l'histoire du programme, mais dans le même temps, il annule le dispositif narratif inventé par Davies (la destruction de Gallifrey) pour expliquer la reconfiguration du monde fictionnel entre les deux séries. Dans un mouvement paradoxal, Moffat présente comme possible un retour à une configuration du monde fictionnel datant de la série classique, tout en le mettant en scène comme une victoire, un événement, un renouveau dans le fond comme dans la forme. Il permet au programme de rebondir en soulignant sa capacité toujours intacte à se renouveler sans fin.

Après les événements de The Time of the Doctor (7.15), le Onzième Docteur, arrivé au terme de son cycle de régénérations, se croit au seuil de la mort, lorsqu'il est sauvé in extremis par les Seigneurs du Temps qu'il a secourus dans l'épisode précédent, et se voit accorder de nouveau la capacité de se régénérer. Peter Capaldi, âgé de 56 ans, succède ainsi à Matt Smith (32 ans), et devient de loin le plus vieux Docteur de la nouvelle série, évoquant les figures de patriarches de la série classique en même temps que l'âge du programme. Sa compagne Clara peine à le reconnaître; dans l'ouverture de la saison 8 ( «Deep Breath », 8.1), via le procédé du voyage dans le temps, le Docteur incarné par Matt Smith adresse à Clara un ultime appel téléphonique ${ }^{38}$, et lui assure que l'homme qui se tient à présent devant elle a beau avoir changé de visage, il est toujours lui-même. Il n'a jamais la même apparence, peine parfois à être reconnu, parce que son identité même est en flux permanent. Matt Hills, dans sa critique dédiée à « Deep Breath »39, notait que la scène, chargée d'émotion, pouvait être perçue comme irrespectueuse des fans,

37 Booth, op. cit.

${ }^{38}$ De la même façon que, dans « The Bells of Saint John » (7.7), c'est elle qui l'appelle «par erreur » avant leur première rencontre, bouclant la boucle.

39 Matt Hills, "Fans can breathe again after Peter Capaldi's captivating Doctor Who debut », in The Conversation, consulté le 3 septembre 2014. 
comme si ce clin d'œil réflexif trahissait un manque de confiance de la part des primo-producteurs envers celles et ceux qui ont suivi la série au travers de ses multiples changements.

Tout comme le Docteur, la nature même du programme est évidemment en flux constant. Doctor Who assume sa multiauctorialité, laquelle fait partie de l'image du programme malgré une communication parfois centrée sur la seule figure du head writer ou d'un.e producteur.ice. L'univers étendu, elliptique, centrifuge, rhizomique, a gagné en cohérence pour les célébrations du cinquantenaire, les éditeurs affiliés coordonnant leurs efforts pour proposer des collections spéciales ${ }^{40}$. Reste à voir comment, dans un avenir proche, la BBC choisira d'embrasser ce vaste monde fictionnel, dont la dynamique est intersticielle par essence ; monde fictionnel qui, comme le montre l'épisode-événement du cinquantenaire, ne cesse, à l'image du phénix, de se nourrir de lui-même, de renaître de ses cendres, de rester fidèle à lui-même, quelle que soit son apparence.

\section{Bibliographie}

Bоотн Paul, «Periodising Doctor Who », Science Fiction Film and Television, 7.2, 2014, p. 195-215.

BRITTON Pierce, "It's All New Doctor Who: Authorising New Design and Redesign in the Steven Moffat Era ", Doctor Who, The Eleventh Hour, Andrew O'Day (éd.), Londres, New York, IB Tauris, 2014.

CHAPMAN James, Inside the TARDIS: The Worlds of Doctor Who, Londres, New York, IB Tauris, 2013 (édition révisée).

Collins Frank, «Monsters Under the Bed: Gothic and Fairy-Tale Storytelling in Steven Moffat's Doctor Who ", Doctor Who, The Eleventh Hour, Andrew O’Day (éd.), Londres, New York, IB Tauris, 2014

Davies Russel T., Cook Benjamin, The Writer's Tale: The Final Chapter, Londres, BBC Books, 2010.

Deleuze Gilles, GuATTARI Félix, Capitalisme et schizophrénie : Tome 2, Mille plateaux, Paris, Éditions de Minuit, 1980.

${ }^{40}$ Matt Hills, « Anniversary Adventures in Space and Time: The Changing Faces of Doctor Who's Commemoration ", in Matt Hills, New Dimensions of Doctor Who, Londres, New York, I.B. Tauris, 2013, p. 229. 
HiLls Matt, Triumph of a Time Lord, Londres, New York, I.B. Tauris, 2010.

--, «Torchwood's trans-transmedia: Media tie-ins and brand "fanagement" ", Participations, Vol. 9, No. 2, novembre 2012.

-- (éd.), New Dimensions of Doctor Who, Londres, New York, I.B. Tauris, 2013.

--, " Fans can breathe again after Peter Capaldi's captivating Doctor Who debut ", The Conversation, lien consulté le 3 septembre 2014.

JENkINS Henry, Convergence Culture: Where Old and New Media Collide, New York, New York University Press, 2006.

JoHnson Catherine, "Doctor Who as Programme Brand", New Dimensions of Doctor Who, Matt Hills (éd.), Londres, New York, IB Tauris, 2013.

O'DAY Andrew (éd.), Doctor Who, The Eleventh Hour, Londres, New York, IB Tauris, 2014.

PEARSON Roberta, "The Writer/Producer in American Television ", The Contemporary Television Series, Michael Hamond et Lucy Mazdon (éds.), Édimbourg, Edinburgh University Press, 2005.

PERrYMAn Neil, «"I AM THE DOCTOR !”: Transmedia Adventures in Time and Space ", Doctor Who, The Eleventh Hour, Andrew O'Day (éd.), Londres, New York, IB Tauris, 2014.

PORTER Lynnette, Doctor Who, American Influence, Fan Culture and the Spinoffs, Jefferson, Londres, McFarland \& Company, 2012.

RonAN-Dubois François, " Auteurs, régies discursives et primoproducteurs », Contagions, lien consulté le 3 septembre 2014.

Tulloch John, Alvarado Manuel, Doctor Who: The Unfolding Text, New York, Saint Martin's Press, 1983.

Tulloch John, JENkyns Henry, Science Fiction Audiences: Watching Doctor Who and Star Trek, Londres, New York, Routledge, 1995.

\section{L'auteur}

Florent Favard termine actuellement son doctorat en études cinématographiques à l'université Bordeaux Montaigne sous la direction de Pierre Beylot. Il travaille sur l'unité narrative des séries télévisées de science- 
fiction contemporaines, abordant les notions de clôture narrative, de canonicité, la réception du récit ou encore la configuration des mondes fictionnels. Il s'intéresse plus largement aux évolutions contemporaines du récit sous toutes ses formes et aux genres de l'imaginaire. 